\title{
An Automatic Approach for Document-level Topic Model Evaluation
}

\author{
Shraey Bhatia $^{1} \quad$ Jey Han Lau $^{1,2} \quad$ Timothy Baldwin $^{1}$ \\ ${ }^{1}$ School of Computing and Information Systems, \\ The University of Melbourne \\ ${ }^{2}$ IBM Research \\ shraeybhatia@gmail.com, jeyhan.lauegmail.com, tbeldwin.net
}

\begin{abstract}
Topic models jointly learn topics and document-level topic distribution. Extrinsic evaluation of topic models tends to focus exclusively on topic-level evaluation, e.g. by assessing the coherence of topics. We demonstrate that there can be large discrepancies between topic- and documentlevel model quality, and that basing model evaluation on topic-level analysis can be highly misleading. We propose a method for automatically predicting topic model quality based on analysis of documentlevel topic allocations, and provide empirical evidence for its robustness.
\end{abstract}

\section{Introduction}

Topic models such as latent Dirichlet allocation (Blei et al., 2003) jointly learn latent topics (in the form of multinomial distributions over words) and topic allocations to individual documents (in the form of multinomial distributions over topics), and provide a powerful means of document collection navigation and visualisation (Newman et al., 2010a; Chaney and Blei, 2012; Smith et al., 2017). One property of LDA-style topic models that has contributed to their popularity is that they are highly configurable, and can be structured to capture a myriad of statistical dependencies, such as between topics (Blei and Lafferty, 2006), between documents associated with the same individual (Rosen-Zvi et al., 2004), or between documents associated with individuals in different network relations (Wang and Blei, 2011). This has led to a wealth of topic models of different types, and the need for methods to evaluate different styles of topic model over the same document collections. Test data perplexity is the obvious solution, but it has been shown to correlate poorly with direct human assessment of topic model quality (Chang et al., 2009), motivating the need for automatic topic model evaluation methods which emulate human assessment. Research in this vein has focused primarily on evaluating the quality of individual topics (Newman et al., 2010b; Mimno et al., 2011; Aletras and Stevenson, 2013; Lau et al., 2014; Fang et al., 2016) and largely ignored evaluation of topic allocations to individual documents, and it has become widely accepted that topic-level evaluation is a reliable indicator of the intrinsic quality of the overall topic model (Lau et al., 2014). We challenge this assumption, and demonstrate that topic model evaluation should operate at both the topic and document levels.

Our primary contributions are as follows: (1) we empirically demonstrate that there can be large discrepancies between topic- and document-level topic model evaluation; (2) we demonstrate that previously-proposed document-level evaluation approaches can be misleading, and propose an alternative evaluation method; and (3) we propose an automatic approach to topic model evaluation based on analysis of document-level topic distributions, which we show to correlate strongly with manual annotations.

\section{Related Work}

Perplexity or held-out likelihood has long been used as an intrinsic metric to evaluate topic models (Wallach et al., 2009). Chang et al. (2009) proposed two human judgement tasks, at the topic and document levels, and showed that there is low correlation between perplexity and direct human evaluations of topic model quality. The two tasks took the form of "intruder" tasks, whereby subjects were tasked with identifying an intruder topic word for a given topic, or an intruder topic for a given document. Specifically, in the word intrusion 
task, an intruder word was added to the top-5 topic words, and annotators were asked to identify the intruder word. Similarly in the topic intrusion task, a document and 4 topics were presented - the top-3 topics corresponding to the document and a random intruder topic - and subjects were asked to spot the intruder topic. The intuition behind both methods is that the higher the quality of the topic or topic allocation for a given document, the easier it should be to detect the intruder.

Newman et al. (2010b) proposed to measure topic coherence directly in the form of "observed coherence", in which human judges rated topics directly on an ordinal 3-point scale. They experimented with a range of different methods to automate the rating task, and reported the best results by simply aggregating pointwise mutual information (pmi) scores for different pairings of topic words, based on a sliding window over English Wikipedia.

Building on the work of Chang et al. (2009), Lau et al. (2014) proposed an improved method for estimating observed coherence based on normalised pmi (npmi), and further automated the word intruder detection task based on a combination of word association features (pmi, npmi, CP1, and CP2) in a learn-to-rank model (Joachims, 2006). Additionally, the authors showed a strong correlation between word intrusion and observed coherence, and suggested that it is possible to perform topic model evaluation based on aggregation of word intrusion or observed coherence scores across all topics.

\section{Datasets and Topic Models}

We use two document collections for our experiments: APNEWS and the British National Corpus ("BNC": Burnard (1995)). APNEWS is a collection of Associated Press ${ }^{1}$ news articles from 2009 to 2016, while BNC is an amalgamation of extracts from different sources such as books, journals, letters, and pamphlets. We sample $50 \mathrm{~K}$ and $15 \mathrm{~K}$ documents from APNEWS and BNC, respectively, to create two datasets for our experiments.

In terms of preprocessing, we use Stanford CoreNLP (Manning et al., 2014) to tokenise words and sentences. We additionally remove stop words, ${ }^{2}$ lower-case all word tokens, filter word types which occur less than 10 times, and exclude

\footnotetext{
${ }^{1}$ https: / / www .ap.org/en-gb/

${ }^{2}$ We use Mallet's stop word list: https://github. $\mathrm{com} / \mathrm{mimno} / \mathrm{Mallet} / \mathrm{tree} / \mathrm{master} / \mathrm{stoplists}$
}

the top $0.1 \%$ most frequent word types. Statistics for each of the preprocessed datasets are provided in Table 1.

Similarly to Chang et al. (2009), we base our analysis on a representative selection of topic models, each of which we train over APNEWS and BNC to generate 100 topics:

- Ida (Blei et al., 2003) uses a symmetric Dirichlet prior to model both document-level topic mixtures and topic-level word mixtures. It is one of the most commonly used topic model implementations and serve as a benchmark for comparison. We use Mallet's implementation of $1 \mathrm{da}$ for our experiments. Note that Mallet implements various enhancements to the basic LDA model, including the use of an asymmetric-symmetric prior.

- ctm (Blei and Lafferty, 2006) is an extension of 1 da that uses a logistic normal prior over topic proportions instead of a Dirichlet prior to model correlations between different topics and reduce overlap in topic content.

- hca (Buntine and Mishra, 2014) is an extension to LDA to capture word burstiness (Doyle and Elkan, 2009), based on the observation that there tends to be higher likelihood of generating a word which has already been seen recently. Word generation is modelled by a Pitman-Yor process (Chen et al., 2011).

- ntm (Cao et al., 2015) is a neural topic model, where topic-word multinomials are modelled as a look-up layer of words, and topic-document multinomials are modelled as a look-up layer of documents. The output layer of the network is given by the dot product of the two vectors. There are 2 variants of $n t m$ : unsupervised and supervised. We use only the unsupervised variant in our experiments.

- cluster is a baseline topic model, specifically designed to produce highly coherent topics but "bland" topic allocations. We represent word types in the documents with pre-trained word2vec vectors (Mikolov et al., 2013a,b), pre-trained on Google News, ${ }^{3}$ and create word clusters using $k$-means clustering $(k=100)$ to generate the topics. We derive the multinomial distribution for each topic based on the cosine distance to the cluster centroid, and

\footnotetext{
${ }^{3}$ Available from: https://code.google.com/ archive/word2vec.
} 


\begin{tabular}{ccc}
\hline Dataset & \#Docs & \#Tokens \\
\hline APNEWS & $50 \mathrm{~K}$ & $15 \mathrm{M}$ \\
BNC & $15 \mathrm{~K}$ & $18 \mathrm{M}$ \\
\hline
\end{tabular}

Table 1: Statistics for the two document collections used in our experiments

\begin{tabular}{ccc}
\hline Model & APNEWS & BNC \\
\hline lda & 0.16 & 0.14 \\
ctm & 0.07 & 0.09 \\
hca & 0.14 & 0.08 \\
ntm & 0.10 & 0.08 \\
cluster & 0.18 & 0.17 \\
\hline
\end{tabular}

Table 2: Topic coherence scores (npmi)

linear normalisation across all words.

To generate the topic allocation for a given document, we first calculate a document representation based on the mean of the word2 vec vectors of its content words. For each cluster, we represent them by calculating the mean word2 $\mathrm{vec}$ vectors of its top- 10 words. Given the document vector and clusters/topics, we calculate the similarity of the document to each cluster based on cosine similarity, and finally (linearly) normalise the similarities to generate a probability distribution.

\section{Topic-level Evaluation: Topic Coherence}

Pointwise mutual information (and its normalised variant npmi) is a common association measure to estimate topic coherence (Newman et al., 2010b; Mimno et al., 2011; Aletras and Stevenson, 2013; Lau et al., 2014; Fang et al., 2016). Although the method is successful in assessing topic quality, it tells us little about the association between documents and topics. As we will see, a topic model can produce topics that are coherent - in terms of npmi association - but poor descriptor of the overall concepts in the document collection.

We first compute topic coherence for all 5 topic models over APNEWS and BNC using npmi (Lau et al., 2014) and present the results in Table $2 .{ }^{4} \mathrm{We}$ see that lda and cluster perform consistently well across both datasets. hca performs well over

\footnotetext{
${ }^{4}$ We use the following open source toolkit to compute topic coherence: https://github.com/jhlau/ topic_interpretability.
}

APNEWS but poorly over BNC. Both ctm and ntm topics appear to have low coherence over the two datasets.

Based on these results, one would conclude that cluster is a good topic model, as it produces very coherent topics. To better understand the nature and quality of the topics, we present a random sample of Ida and cluster topics in Table 3.

Looking at the topics, we see that cluster tends to include different inflectional forms of the same word (e.g. prohibited, probihiting) and nearsynonyms/sister words (e.g. river, lake, creeks) in a single topic. This explains the strong npmi association of the cluster topics. On the other hand, I da discovers related words that collectively describe concepts rather than just clustering (near) synonyms. This suggests that the topic coherence metric alone may not completely capture topic model quality, leading us to also investigate the topic distribution associated with documents from our collections.

\section{Human Evaluation of Document-level Topic Allocations}

In this section, we describe a series of manual evaluations of document-level topic allocations, in order to get a more holistic evaluation of the true quality of the different topic models (in line with the original work of Chang et al. (2009)).

\subsection{Topic Intrusion}

The goal of the topic intrusion task is to examine whether the document-topic allocations from a given topic model accord with manual judgements. We formulate the task similarly to Chang et al. (2009), in presenting the human judges with a snippet from each document, along with four topics. The four topics comprise the top-3 highest probability topics related to document, and one intruder topic. Each annotator is required to pick the topic that is least representative of the document, with the expectation that the better the topic model, the more readily they should be able to pick the intruder topic. The intruder topic is sampled randomly, subject to the following conditions: (1) it should be a low probability topic for the target document; and (2) it should be a high probability topic for at least one other document. The first constraint is intended to ensure that the intruder topic is unrelated to the target document, while the second constraint is intended to select a topic that is highly 


\begin{tabular}{cl}
\hline Model & Topics \\
\hline lda & $\begin{array}{l}\text { oil gas drilling gulf spill natural pipeline wells industry energy } \\
\text { computer video screen program text disk windows electronic machine graphics } \\
\text { health care hospital services medical staff patients service child authority }\end{array}$ \\
\hline \multirow{2}{*}{ cluster } & $\begin{array}{l}\text { river creek lake rivers dam tributary lakes reservoir tributaries creeks } \\
\text { prohibited forbid prohibiting prohibits violated prohibit contravened forbids violate barred } \\
\text { terrace courtyard staircase staircases courtyards walls pergola walkway stairways walkways }\end{array}$ \\
\hline
\end{tabular}

Table 3: Example lda and cluster topics.

\begin{tabular}{ccc}
\hline \multirow{2}{*}{ Topic Model } & \multicolumn{2}{c}{ Mean Model Precision } \\
\cline { 2 - 3 } & APNEWS & BNC \\
\hline lda & 0.84 & 0.66 \\
ctm & 0.64 & 0.66 \\
hca & 0.60 & 0.44 \\
ntm & 0.26 & 0.17 \\
cluster & 0.39 & 0.48 \\
\hline
\end{tabular}

Table 4: Mean model precision for human judgements

associated with some documents, and hence likely to be coherent and not a junk topic. Each topic is represented by its top-10 most probable words, and the target document is presented in the form of the first three sentences, with an option to view more of the document if further context is needed.

We used Amazon Mechanical Turk to collect the human judgements, with five document-topic combinations forming a single HIT, one of which acts as a quality control. The control items were sourced from an earlier annotation task where subjects were asked to score the top-5 topics for a target document on a scale of $0-3$. The 50 topscoring documents from this annotation task, with their top-3 topics, were chosen as controls. The intruder topic for the control was generated by randomly selecting 10 words from the corpus vocabulary. In order to pass quality control, each worker had to correctly select the intruder topic for the control document-topic item over $60 \%$ of time (across all HITs they completed). Each document-topic pair was rated by 10 annotators initially, and for HITs where less than 3 annotations passed quality control, we reposted them for a second round of annotation.

For our annotation task, we randomly sampled 100 documents from each of our two datasets, for

\begin{tabular}{ccc}
\hline \multirow{2}{*}{ Topic Model } & \multicolumn{2}{c}{ Mean Topic Log Odds } \\
\cline { 2 - 3 } & APNEWS & BNC \\
\hline lda & -0.78 & -1.84 \\
Ctm & -1.04 & -1.60 \\
hca & -2.09 & -3.61 \\
ntm & -7.16 & -6.32 \\
cluster & -0.12 & -0.10 \\
\hline
\end{tabular}

Table 5: Mean topic log odds for human judgements

each of which we generate document-topic items based on the five different topic models. In total, therefore, we annotated 1000 (100 documents $\times 2$ collections $\times 5$ topic models) document-topic combinations. After quality control, the final dataset contains an average of 5.4 and 5.5 valid intruder topic annotations for APNEWS and BNC, respectively.

Chang et al. (2009) proposed topic log odds ("TLO") as a means of evaluating the topic intrusion task. The authors defined topic log odds for a document-topic pair as the difference in the logprobability assigned to the intruder and the logprobability assigned to the topic chosen by a given annotator, which they then averaged across annotators to get a TLO score for a single document. Separately, Chang et al. (2009) proposed model precision as a means of evaluating the word intrusion task, whereby they simply calculated the proportion of annotators who correctly selected the intruder word for a given topic. In addition to presenting results based on TLO, we apply the model precision methodology in our evaluation of the topic intrusion task, in calculating the proportion of annotators who correctly selected the intruder topic for a given document, which we then average across documents to derive a model score. 


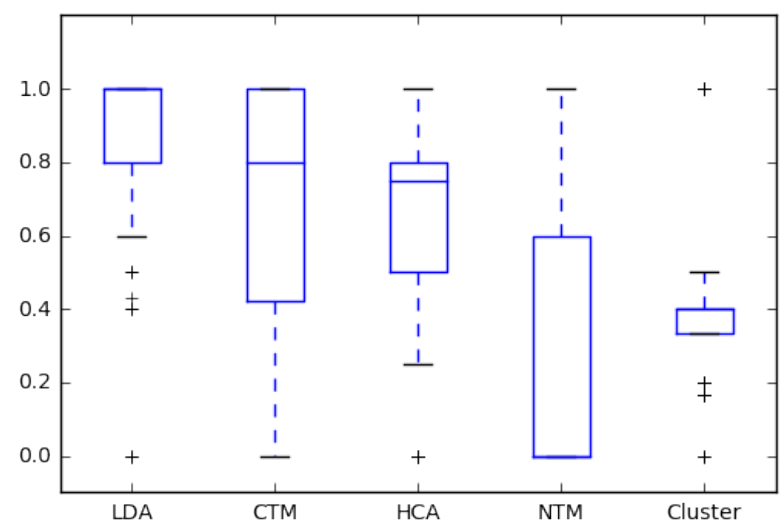

(a) APNEWS

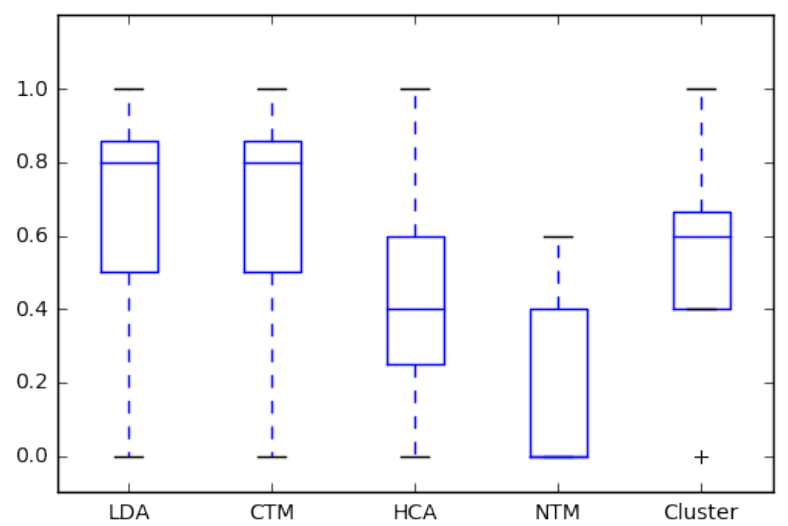

(b) BNC

Figure 1: Boxplots of model precision

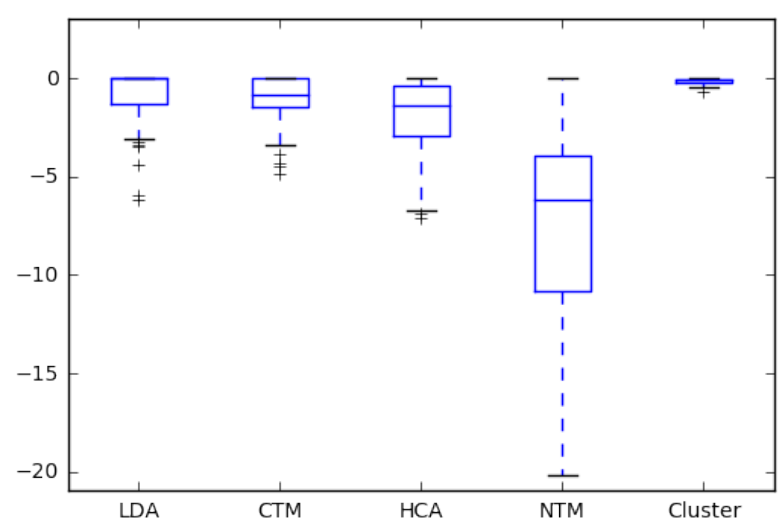

(a) APNEWS

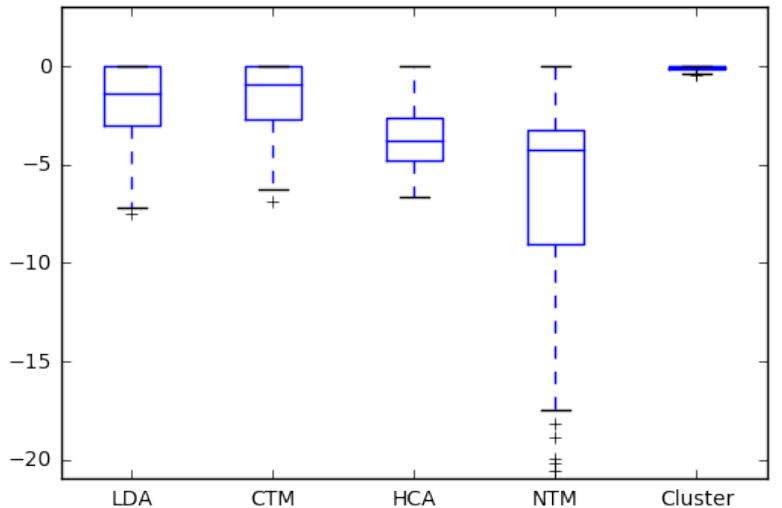

(b) BNC

Figure 2: Boxplots of topic log odds

The results of the human annotation task are summarised in Tables 4 and 5. Looking at model precision for APNEWS first, we see that lda outperforms the other topic models. ctm and hca perform credibly, whereas ntm and cluster are quite poor. Moving on to BNC, we see a drop in score for $1 \mathrm{da}$, to a level comparable with ctm. cluster improves slightly higher than BNC, whereas hca drops considerably (despite being designed specifically to deal with word burstiness in the longer documents characteristic of BNC). Figure 1 shows boxplots for topic-level model precision, and reflects a similar trend.

Looking next to TLO in Table 5, we see a totally different picture, with cluster being rated as the best topic model by a clear margin. This exposes a flaw in the TLO formulation, in the case of adversarial topic models such as cluster which assign near-uniform probabilities across all topics. This results in the difference in probability mass being very close to the upper bound of zero in all cases, meaning that even for random topic selection, TLO is near perfect. We can also see this in Figure 2, where the boxes for cluster have nearly zero range. Indeed, if we combined the results for TLO with those for topic coherence, we would (very wrongly!) conclude that cluster performs best over both document collections. More encouragingly, for the other four topic models, the results for TLO are much more consistent with those based on model precision.

\subsection{Direct Annotation of Topic Assignments}

Newman et al. (2010b) proposed a more direct approach to topic coherence, by asking people to rate topics directly based on the top- $N$ words. Taking inspiration from their methodology, we propose to directly annotate each topic assigned to a target document. We present the human annotators with the target document and the top-ranked (high- 


\begin{tabular}{ccc}
\hline \multirow{2}{*}{ Topic Model } & \multicolumn{2}{c}{ Average rating } \\
\cline { 2 - 3 } & APNEWS & BNC \\
\hline lda & 1.26 & 1.01 \\
ctm & 0.96 & 1.02 \\
hca & 0.95 & 0.90 \\
ntm & 0.36 & 0.46 \\
cluster & 0.41 & 0.66 \\
\hline
\end{tabular}

Table 6: Top-1 document-topic rating for each topic model

est probability) topic from each of the five topic models, and ask them to rate each topic on an ordinal scale of $0-3$. At the model level, we take the mean rating over all document-topic pairings for that topic model (based, once again, on 100 documents per collection). ${ }^{5}$ We summarise the findings in Table 6.

We observe that, in the case of APNEWS, Ida does considerably better than $\mathrm{ctm}$ and hca, whereas for BNC, Ida and ctm are quite close, with hca close behind. cluster and ntm do poorly across both datasets. The overall trend for APNEWS of lda $>$ ctm $>$ hca $>$ cluster $>$ $\mathrm{ntm}$ is consistent with the model precision results in Table 4. In the case of BNC, the observation of ctm $\approx 1$ da $>$ hca $>$ cluster $>$ ntm is also broadly the same, except that hca does not do as well over the topic intrusion task. Here, we are more interested in the relative performance of topic models than absolute numbers, although the low absolute scores are an indication that it is a difficult annotation task.

Broadly combined across the two evaluation methodologies, lda and ctm are top-performing, hca gets mixed results, and cluster and ntm are the lowest performers. These results generally agree with the model precision findings, demonstrating that model precision is a more robust metric than TLO.

\section{Automatic Evaluation}

A limitation of the topic intrusion task is that it requires manual annotation, making it ill-suited for large-scale or automatic evaluation. We present the first attempt to automate the prediction of the intruder topic, with the aim of developing an approach to topic model evaluation which comple-

\footnotetext{
${ }^{5}$ The 100 documents used for this task were different to the ones used in Section 5.1.
}

ments topic coherence (as motivated in Sections 4 and 5).

\subsection{Methodology}

We build a support vector regression (SVR) model (Joachims, 2006) to rank topics given a document to select the intruder topic. We first explain an intuition of the features that are driving the SVR.

To rank topics for a document, we need to first compute the probability of a topic $t$ given document $d$, i.e. $P(t \mid d)$. We can invert the condition using Bayes rule:

$$
\begin{aligned}
P(t \mid d) & =\frac{P(d \mid t) P(t)}{P(d)} \\
& \propto P(d \mid t) P(t)
\end{aligned}
$$

We can omit $P(d)$ as the probability of document $d$ is constant for the topics that we are ranking.

Next we represent topic $t$ using its top- $N$ highest probability words, giving:

$$
\begin{aligned}
P(t \mid d) \propto P\left(d \mid w_{1}, \ldots, w_{N}\right) P\left(w_{1}, \ldots, w_{N}\right) & \propto \log P\left(d \mid w_{1}, \ldots, w_{N}\right)+ \\
& \log P\left(w_{1}, \ldots, w_{N}\right)
\end{aligned}
$$

The first term $\log P\left(d \mid w_{1}, \ldots, w_{N}\right)$ can be interpreted from an information retrieval perspective, where we are computing the relevance of document $d$ given query terms $w_{1}, w_{2}, \ldots, w_{N}$. This term constitutes the first feature for the SVR. We use Indri ${ }^{6}$ to index the document collection, and compute $\log P\left(d \mid w_{1}, \ldots, w_{N}\right)$ given a set of query words and a document. ${ }^{7}$

We estimate the second term, $\log P\left(w_{1}, \ldots, w_{N}\right)$, using the pairwise probability of the topic words:

$$
\sum_{0<i \leq m} \sum_{i+1 \leq j \leq m} \log \frac{\#\left(w_{i}, w_{j}\right)}{\#(\cdot)}
$$

where $m$ denotes the number of topic words used, $\#\left(w_{i}, w_{j}\right)$ is the number of documents where word $w_{i}$ and $w_{j}$ co-occur, and \#(.) is the total number of documents. We explore using two values of $m$ here: 5 and $10 .{ }^{8}$ These two values constitute the second and third features of the SVR.

To train the SVR, we sample 1700 random documents and split them into 1600/100 documents for the training and test partitions, respectively.

\footnotetext{
${ }^{6}$ http: / / www. lemurproject.org

${ }^{7} N=10$.

${ }^{8}$ That is, if $m=5$, we compute pairwise probabilities using the top- 5 topic words.
} 


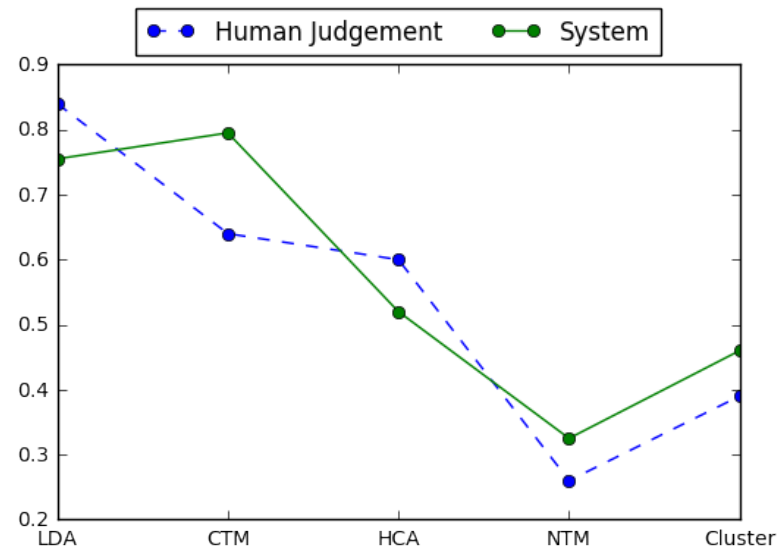

(a) APNEWS

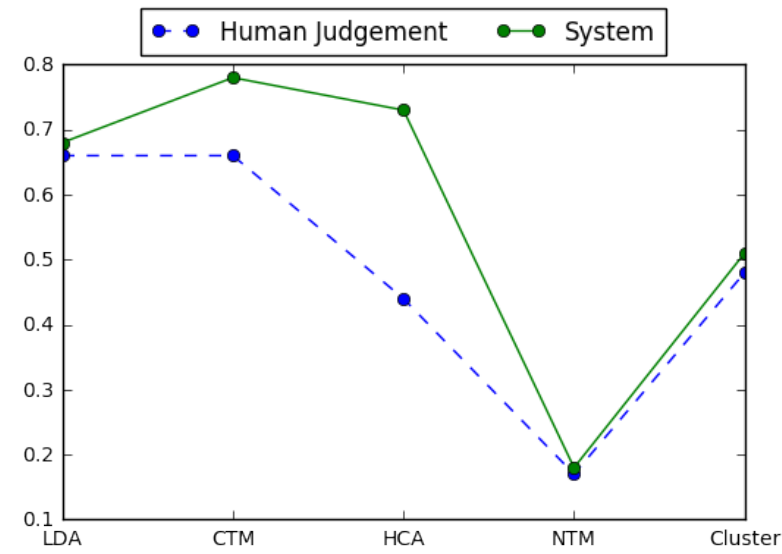

(b) BNC

Figure 3: Mean Model Precision Comparison

The test documents are the same 100 documents that were previously used for intruder topics (Section 5.1). As the intruder topics are artificially generated, we can sample additional documents to create a larger training set for the SVR; the ability to generate arbitrary training data is a strength of our method.

We pool together all 5 topic models when training the SVR, thereby generating 8000 training and 500 development and testing instances for each dataset. For each document, the SVR is trained to rank the topics in terms of their likelihood of being an intruder topic. ${ }^{9}$ The top-ranking topic is selected as the system-predicted intruder word, and model precision is computed as before (Section 5.1). ${ }^{10}$

\subsection{System results}

In Figure 3, we present the human vs. system mean model precision on the test partition for each of the topic models. We see that the trend line for the system model precision very closely tracks that of human model precision. In general, the best systems - Ida and ctm - and the worst systems — ntm and cluster — are predicted correctly. The correlation between the two is very high, at $r=0.88$ and 0.87 for APNEWS and BNC, respectively. This suggests that the automated method is a reliable means of evaluating document-level topic model quality.

\footnotetext{
${ }^{9}$ We use the default hyper-parameter values for the SVR $(C=0.01)$, and hence do no require a development set for tuning.

${ }^{10}$ Note that the system model precision for each documenttopic combination is a binary value as there is only 1 system — as opposed to multiple annotators - selecting an intruder word.
}

\section{Discussion}

To better understand the differences between human- and system-predicted intruder topics, we present a number of documents and their associated topics in Table 7, focusing specifically on: (a) intruder topics that humans struggle to identify but our automatic method reliably detects; and (b) conversely, intruder topics which humans readily identify but our method struggles to detect.

Looking at the topics across the two types of errors, we notice that there are often multiple "bad" topics for these documents: occasionally the annotators are able to single out the worst topic while the system fails (1st and 2nd document), but sometimes the opposite happens (3rd and 4th document). In the first case, the top-ranking topic (church, gay, ...) from the topic model is associated with the document because of the service, but actually capturing a very different aspect of religion to what is discussed in the document, which leads our method astray. A similar effect is seen with the second document. In the case of the third and fourth documents, there is actually content further down in the document which is relevant to the topics the human annotators select, but it is not apparent in the document snippet presented to the annotators. That is, the effect is caused by resource limitations for the annotation task, that our automated method does not suffer from.

When we aggregate the top-level model precision values for a topic model, these differences are averaged out (hence the strong correlation in Section 6.2), but these qualitative analyses reveal that there are still slight disparities between human 


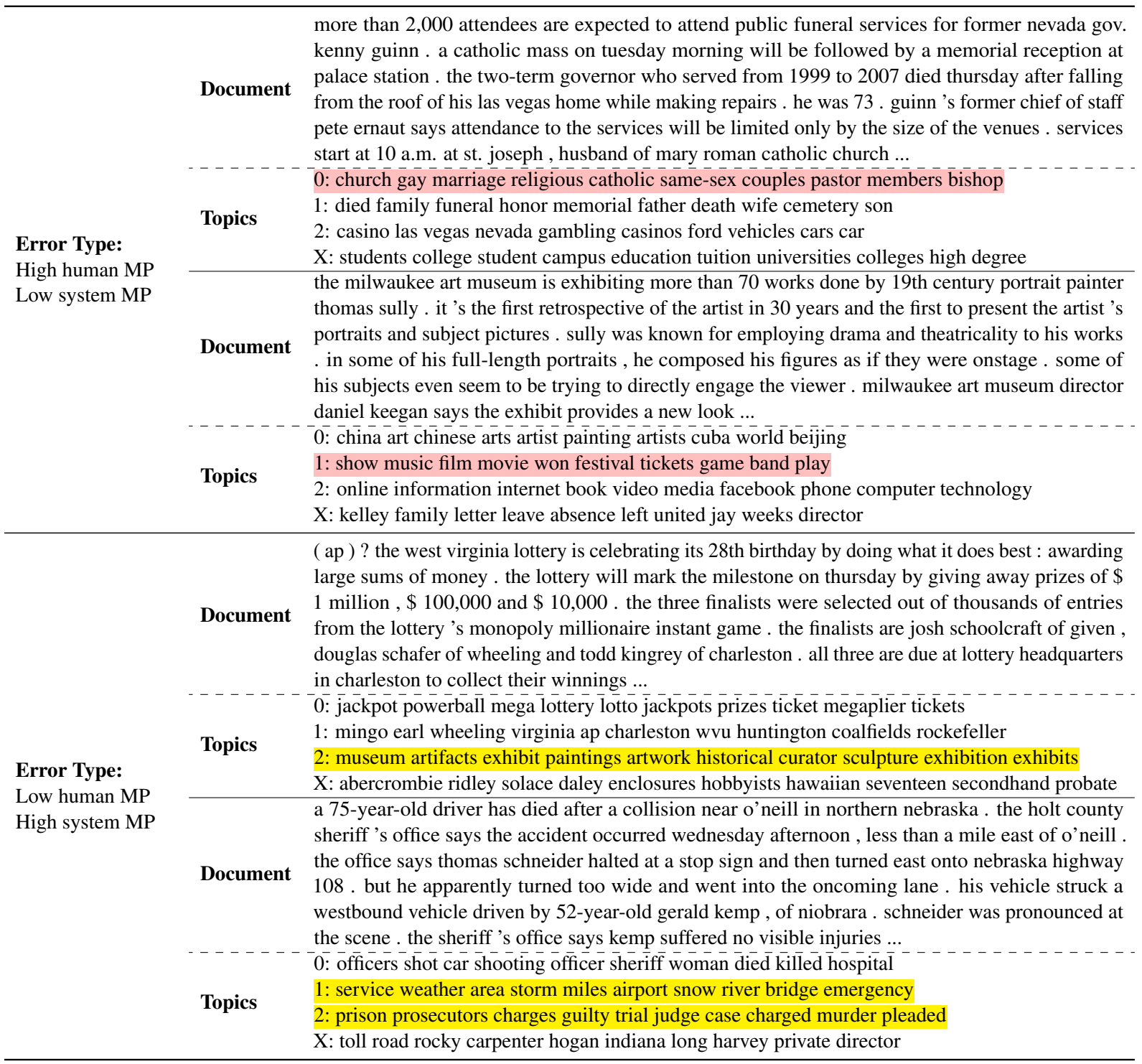

Table 7: Document and topic examples for two types of errors. "MP" denotes model precision, "X" the intruder topic, and the indices the ranking of the topics. Topics highlighted in pink (yellow) are those incorrectly selected by the system (humans) as intruder topics.

annotators and the automated method in intruder topic selection.

To further understand how the topics relate to the documents in different topic models, we present documents with the corresponding topics for different topic models in Table 8.

In the human annotation task, we use the top-10 most probable words to represent a topic. We use 10 words as it is the standard approach to visualising topics, but this is an important hyper-parameter which needs to be investigated further (Lau and Baldwin, 2016), which we leave to future work.

\section{Conclusion}

We demonstrate empirically that there can be large discrepancies between topic coherence and document-topic associations. By way of designing an artificial topic model, we showed that a topic model can simultaneously produce topics that are coherent but be largely undescriptive of the document collection. We propose a method to automatically predict document-level topic quality and found encouraging correlation with manual evaluation, suggesting that it can be used as an alternative approach for extrinsic topic model evaluation. 


\begin{tabular}{|c|c|c|}
\hline lda & $\begin{array}{l} \\
\text { Topics }\end{array}$ & $\begin{array}{l}\text { more than } 2,000 \text { attendees are expected to attend public funeral services for former nevada gov. } \\
\text { kenny guinn . a catholic mass on tuesday morning will be followed by a memorial reception at } \\
\text { palace station . the two-term governor who served from } 1999 \text { to } 2007 \text { died thursday after falling } \\
\text { from the roof of his las vegas home while making repairs . he was } 73 \text {. guinn 's former chief of staff } \\
\text { pete ernaut says attendance to the services will be limited only by the size of the venues . services } \\
\text { start at } 10 \text { a.m. at st. joseph , husband of mary roman catholic church ... } \\
\text { 0: church gay marriage religious catholic same-sex couples pastor members bishop } \\
\text { 1: died family funeral honor memorial father death wife cemetery son }\end{array}$ \\
\hline hca & $\begin{array}{l}\text { Document } \\
\text { Topics }\end{array}$ & $\begin{array}{l}\text { usa today founder al neuharth has died in cocoa beach, florida . he was } 89 \text {. the news was announced } \\
\text { friday by usa today and by the newseum, which he also founded . neuharth changed american } \\
\text { newspapers by putting easy-to-read articles and bright graphics in his national daily publication, } \\
\text { which he began in } 1982 \text { when he ran the gannett co. newspaper group . he wanted to create a bright } \\
\text {, breezy, fun newspaper that would catch people 's attention and not take itself too seriously. its } \\
\text { annual revenues increased from } 200 \text { million to more than } 3 \text { billion ... } \\
\text { 0: } \text { honorary commencement philanthropist journalism distinguished honored bachelor pulitzer }_{\text {doctorate harvard }}^{-} \\
\text {1: shortfall premiums budget reductions cuts shortfalls salaries pensions revenues budgets }\end{array}$ \\
\hline ctm & Topics & $\begin{array}{l}\text { a teenage driver who survived a southeastern indiana crash that killed three other youths will spend } \\
90 \text { days in juvenile detention and surrender his driver 's license until age } 21 \text {. the } 17 \text {-year-old driver } \\
\text { admitted to charges of reckless homicide and reckless driving during a ripley county juvenile court } \\
\text { hearing thursday in versailles, indiana state police sgt. noel houze jr. told the associated press . the } \\
\text { teenager choked back sobs throughout the half-hour hearing . the teen will be sent to a juvenile } \\
\text { facility in muncie. he also must complete } 350 \text { hours of community service... } \\
\text { 0: officers shot car shooting officer sheriff woman died killed hospital } \\
\text { 1: prison prosecutors charges guilty trial judge case charged murder pleaded }\end{array}$ \\
\hline ntm & Document & $\begin{array}{l}\text { a judge in will county has approved further testing on the coat an oswego man was wearing when } \\
\text { his wife and three children were found shot to death in } 2007 \text {. christopher vaughn is accused } \\
\text { of killing his family inside their suv, which was parked on a frontage road along interstate } 55 \text {. } \\
\text { authorities found kimberly vaughn shot to death, along with their children, 12-year-old abigayle, } \\
11 \text {-year-old cassandra and 8-year-old blake . assistant state 's attorney mike fitzgerald on monday } \\
\text { said prosecutors asked for more dna testing on the coat ... } \\
0 \text { : arraigned burglarizing arrested bigamy detectives motorcyclist arraignment coroner accomplice } \\
\text { fondled } \\
\text { 1: quarterly pretax dividend profit annualized earnings profits stockholders writedown premarket }\end{array}$ \\
\hline cluster & Document & $\begin{array}{l}\text { a southwest idaho district court judge has been arrested on suspicion of misdemeanor driving under } \\
\text { the influence . the idaho press-tribune reports ( http://bit.ly/npiita ) that 3rd district court judge } \\
\text { renae hoff was taken into custody early saturday morning in meridian . meridian deputy police chief } \\
\text { tracy basterrechea says an officer pulled the } 61 \text {-year-old hoff over after she failed to " maintain the } \\
\text { lane of travel." } \\
\text { 0: suppliers manufacturers companies importers supplier exporters distributors market wholesalers } \\
\text { export } \\
\text { 1: deported deportation incarcerated prison detention jail parole imprisoned convicts incarceration }\end{array}$ \\
\hline
\end{tabular}

Table 8: Example documents and their corresponding topics for different topic models

\section{Acknowledgements}

This research was supported in part by the Australian Research Council.

\section{References}

Nikos Aletras and Mark Stevenson. 2013. Evaluating topic coherence using distributional semantics. In Proceedings of the Tenth International Workshop on Computational Semantics (IWCS-10). Potsdam, Germany, pages 13-22.

David Blei and John Lafferty. 2006. Correlated topic models. Advances in Neural Information Processing Systems 18.

David M Blei, Andrew Y Ng, and Michael I Jordan. 2003. Latent Dirichlet allocation. Journal of Machine Learning Research 3:993-1022.

Wray L Buntine and Swapnil Mishra. 2014. Experiments with non-parametric topic models. In Proceedings of the 20th ACM SIGKDD International Conference on Knowledge Discovery and Data Mining. pages 881-890.

Lou Burnard. 1995. User guide for the British National Corpus.

Ziqiang Cao, Sujian Li, Yang Liu, Wenjie Li, and Heng 
Ji. 2015. A novel neural topic model and its supervised extension. In Proceedings of AAAI 2015. pages 2210-2216.

Allison June-Barlow Chaney and David M. Blei. 2012. Visualizing topic models. In Proceedings of the 6th International Conference on Weblogs and Social Media (ICWSM 2012). Dublin, Ireland.

Jonathan Chang, Sean Gerrish, Chong Wang, Jordan L. Boyd-Graber, and David M. Blei. 2009. Reading tea leaves: How humans interpret topic models. In Advances in Neural Information Processing Systems 21 (NIPS-09). Vancouver, Canada, pages 288-296.

Changyou Chen, Lan Du, and Wray Buntine. 2011. Sampling table configurations for the hierarchical poisson-dirichlet process. Machine Learning and Knowledge Discovery in Databases pages 296-311.

Gabriel Doyle and Charles Elkan. 2009. Accounting for burstiness in topic models. In Proceedings of the 26th Annual International Conference on Machine Learning. pages 281-288.

Anjie Fang, Craig Macdonald, Iadh Ounis, and Philip Habel. 2016. Using word embedding to evaluate the coherence of topics from Twitter data. In Proceedings of the 39th International ACM SIGIR conference on Research and Development in Information Retrieval. pages 1057-1060.

Thorsten Joachims. 2006. Training linear SVMs in linear time. In Proceedings of the 12th ACM SIGKDD International Conference on Knowledge Discovery and Data Mining. pages 217-226.

Jey Han Lau and Timothy Baldwin. 2016. The sensitivity of topic coherence evaluation to topic cardinality. In Proceedings of NAACL-HLT . pages 483-487.

Jey Han Lau, David Newman, and Timothy Baldwin. 2014. Machine reading tea leaves: Automatically evaluating topic coherence and topic model quality. In Proceedings of EACL 2014. pages 530-539.

Christopher D. Manning, Mihai Surdeanu, John Bauer, Jenny Finkel, Steven J. Bethard, and David McClosky. 2014. The Stanford CoreNLP natural language processing toolkit. In Association for Computational Linguistics (ACL) System Demonstrations. Baltimore, USA, pages 55-60.

Tomas Mikolov, Kai Chen, Greg Corrado, and Jeffrey Dean. 2013a. Efficient estimation of word representations in vector space. In Proceedings of Workshop at the International Conference on Learning Representations, 2013. Scottsdale, USA.

Tomas Mikolov, Ilya Sutskever, Kai Chen, Greg S Corrado, and Jeff Dean. 2013b. Distributed representations of words and phrases and their compositionality. In Advances in Neural Information Processing Systems. pages 3111-3119.
David Mimno, Hanna Wallach, Edmund Talley, Miriam Leenders, and Andrew McCallum. 2011. Optimizing semantic coherence in topic models. In Proceedings of the 2011 Conference on Empirical Methods in Natural Language Processing (EMNLP 2011). Edinburgh, UK, pages 262-272.

David Newman, Timothy Baldwin, Lawrence Cavedon, Sarvnaz Karimi, David Martinez, and Justin Zobel. 2010a. Visualizing document collections and search results using topic mapping. Journal of Web Semantics 8(2-3):169-175.

David Newman, Jey Han Lau, Karl Grieser, and Timothy Baldwin. 2010b. Automatic evaluation of topic coherence. In Human Language Technologies: The 2010 Annual Conference of the North American Chapter of the Association for Computational Linguistics. pages 100-108.

Michal Rosen-Zvi, Thomas Griffiths, Mark Steyvers, and Padhraic Smyth. 2004. The author-topic model for authors and documents. In Proceedings of the 20th Conference on Uncertainty in Artificial Intelligence. pages 487-494.

Alison Smith, Tak Yeon Lee, Forough PoursabziSangdeh, Jordan Boyd-Graber, Kevin Seppi, Niklas Elmqvist, and Leah Findlater. 2017. Evaluating visual representations for topic understanding and their effects on manually generated labels. Transactions of the Association for Computational Linguistics 5:1-15.

Hanna M Wallach, Iain Murray, Ruslan Salakhutdinov, and David Mimno. 2009. Evaluation methods for topic models. In Proceedings of the 26th International Conference on Machine Learning (ICML 2009). Montreal, Canada, pages 1105-1112.

Chong Wang and David M. Blei. 2011. Collaborative topic modeling for recommending scientific articles. In Proceedings of the 17th ACM SIGKDD International Conference on Knowledge Discovery and Data Mining. pages 448-456. 